\title{
Método Doman para la inclusión e intervención de niños con Trastorno del Desarrollo del Lenguaje.
}

\section{Doman Method for the inclusion and language intervention of children with language development disorder.}

\author{
Alba Ayuso Lanchares \\ Facultad de Medicina. Universidad de Valladolid (España) \\ alba.ayuso@uva.es \\ Rosa Belén Santiago Pardo \\ Facultad de Medicina. Universidad de Valladolid (España) \\ santiago@med.uva.es \\ Inés Ruiz Requies \\ Facultad de Educación y Trabajo Social. Universidad de Valladolid (España) \\ inesrure@pdg.uva.es
}

Fecha recepción: 22/07/2019

Páginas 91-105

Fecha aceptación: 25/11/2019

\section{Resumen.}

Los bits de inteligencia del Método Doman son una secuencia de dibujos o fotografías, acompañadas por la palabra escrita de lo que representan, que se les va mostrando a los niños. Existe mucha controversia sobre este método. El objetivo de este trabajo es comprobar la eficacia de la aplicación de los bits de inteligencia en niños con trastornos del desarrollo del lenguaje (TDL). El vacío científico sobre el tema provoca desconocimiento sobre su efectividad en los tratamientos logopédicos. Esta investigación sigue un método de investigación cuantitativo preexperimental de medidas repetidas pre-post en el cual hay un único grupo. Los resultados preliminares indican que los participantes mejoran en los niveles fonético- fonológico, pero no se encuentran mejoras en otros niveles. Al no revelarse mejoras significativas en los otros niveles, se recomienda adaptar la metodología de aplicación de los bits de inteligencia para utilizarlos en sucesivas sesiones dentro de la escuela, eliminar la excesiva responsabilidad de la familia durante la intervención, aumentar el número de palabras en cada pase y, finalmente, sustituir las palabras mostradas adecuándolas al uso normo típico del desarrollo del lenguaje y la edad del niño.

Palabras clave: retraso; lenguaje; método educativo; intervención, Doman

\section{Abstract.}

The Doman flashcards of the Doman Method are a sequence of drawings or photographs, accompanied by the written word of what they represent, which is shown to the children. There is much controversy about this method. The aim of this work is to verify the effectiveness of the application of Doman flashcards in children with 
language disorders. The scientific vacuum on this topic causes ignorance about their effectiveness in speech therapy treatments. This research follows a pre-experimental quantitative research method of repeated pre-post measures in which there is a single group. The preliminary results indicate that the participants improve in the phoneticphonological levels, but no improvements are found in other levels. When no significant improvements are revealed in the other levels, it is recommended to adapt the application methodology of flashcards method to be used in successive sessions within the school, to eliminate the excessive responsibility of the family during the intervention, to increase the number of words in each pass and, finally, substitute the words shown, adapting them to the norm typical use of language development and the child's age.

Keywords: delay; language; education methodology; intervention, Doman.

\section{1.-Introducción.}

El método Glenn Doman y Carl Decanato -también llamado "Método Doman"- es un método de estimulación temprana. El primer objetivo de Doman cuando lo creó fue conseguir que los niños y niñas -en adelante se utilizará el genérico niños- con lesiones cerebrales graves mejoraran de forma global, tanto física como cognitivamente. Con el tiempo, Doman amplió los posibles destinatarios del método añadiendo a niños sanos con el fin fortalecer su desarrollo intelectual y su potencial (Doman y Doman, 1998).

Este método se subdivide en diferentes programas: programa de lectura, música, matemáticas, lectoescritura, físico y, por último, los bits de inteligencia. Doman defendía el uso simultáneo de estos programas. La aplicación consistía en que, dos veces al año, los padres con el niño asistieran durante una semana completa, al Instituto para el Logro del Potencial Humano, creado por Doman y donde realizó una labor publicitaria y de intervención masiva de su método. Durante ese tiempo, se evaluaba al niño y a sus padres, se les formaba para que continuaran con los programas en casa. Doman consideraba muy importante que los padres se esforzaran y llevaran a cabo todos los ejercicios en casa con su ayuda (Doman y Doman, 1998). El programa de desarrollo del lenguaje que detalla Doman conlleva una serie de principios básicos de la buena enseñanza. Doman y Doman (1998) sugiere utilizar los bits de inteligencia teniendo en cuenta las siguientes recomendaciones: realizar una estimulación temprana, empezar cuando el niño sea lo más pequeño posible, mostrar alegría constante, respetar a los niños teniendo total confianza en ellos, llevar a cabo el programa únicamente cuando los padres y los niños estén contentos, preparar un entorno favorable para el aprendizaje, detener la actividad antes de que el niño desee dejarla, presentar frecuentemente bits nuevos siendo organizados y constantes, no evaluar ni examinar al niño y, tener la precaución de preparar antes de cada sesión el material (Doman y Doman, 1998, p.174).

Por su parte, Doman y Aisen (2012) definen los bits como "un dibujo o una ilustración muy precisa o una fotografía de excelente calidad. Tiene ciertas características muy importantes: debe ser preciso; diferenciado; exacto y nuevo. También tiene que ser grande y claro" (p. 70). Estas láminas, además de mostrar el dibujo, también incluyen la palabra escrita en color rojo, ya que es el color que mejor ven los bebés. Los bits de 
inteligencia están diseñados para aumentar el conocimiento de los bebés y favorecer su maduración neurológica. Las pautas de procedimiento varían dependiendo de la fase de realización y de la edad de los niños, desde mostrar las imágenes con una frecuencia máxima de diez veces al día, bajando la frecuencia según los niños sean más mayores. Los bits se agruparán por categorías, enseñando las láminas de forma rápida, además de decir el nombre de lo que representa en voz alta (Doman y Doman, 1998).

Es un método que no está exento de controversia, ya que en varias publicaciones ha sido desacreditado por la comunidad científica, alegando que carece de evidencias y fundamentos empíricos (p.e. American Academy of Pediatrics, (1999). Además de la falta de evidencia científica, también encontramos otras razones por las que el uso del método preocupa a diferentes sectores científicos como, por ejemplo, la idoneidad de la función rehabilitadora que ejercen los propios padres, debido a que el método exige demasiado a la familia y se pueden descuidar otras necesidades familiares; también preocupa que aumente la ansiedad de unos padres agobiados y confundidos (American Academy for Cerebral Palsy et al., 1968).

Después de estas desacreditaciones, Doman siguió escribiendo libros sobre la efectividad del método en niños con multitud de problemas del neurodesarrollo, como son la parálisis cerebral, epilepsia, espina bífida, déficits sensoriales, diversidad funcional intelectual, autismo y problemas de deglución. El Instituto para el Logro del Potencia Humano, sigue impartiendo cursos y tratando a familias y a niños con problemas, además de continuar publicando artículos que avalan la eficacia del método.

Recientemente, si nos centramos en los resultados del método obtenidos en el lenguaje, que es el área que nos interesa en este artículo, la investigación de Von et al. (2013) dictamina que, empleando los bits de inteligencia, encuentra ciertas mejoras en población con parálisis cerebral, síndromes genéticos, retraso del desarrollo, daño cerebral adquirido y niños con epilepsia, obteniendo una ganancia media en la comprensión del lenguaje de ocho meses, tras dos años de tratamiento.

En otros contextos, como en el educativo, se sigue llevando a cabo en muchos centros educativos de España y de otros países, con el objetivo de que los niños realicen los hitos de desarrollo antes de lo esperado (leer, hablar...), aunque ésta no fuera la idea principal con la que se creó el método; y, además, para lograr una inclusión real de los niños con TDL dentro del aula ordinaria por el tutor, al pretender mejorar su nivel lingüístico con los bits de inteligencia. Además de utilizarlo los tutores también lo utilizan los maestros de audición y lenguaje y los logopedas en sus sesiones de rehabilitación, siendo incluso comercializado en varias páginas de venta de material logopédico. En el caso del logopeda o del maestro de audición y lenguaje, el uso es diferente puesto que este método se suele adaptar a las necesidades de cada niño, vetando a la familia el protagonismo. Esta adaptación se realiza bajo el criterio del propio maestro, debido a que no se encuentra literatura sobre este tema. Este vacío lo observamos cuando se realiza una búsqueda exhaustiva en dos buscadores Scopus y Web Of Science (WOS).

En Scopus, cuando aplicamos las palabras clave "Doman Method" encontramos únicamente 5 resultados (1984, 2011, 2012 y 2018), estos cinco artículos se centran 
sobre todo en patrones motores y no tanto del lenguaje. En caso de añadir la palabra "Language" a los resultados anteriores, no obtenemos resultados.

Realizando la misma búsqueda en otro buscador, WOS (Web of Science), al añadir "Doman method" encontramos 61 resultados. Con el objetivo de delimitar los resultados, eliminamos los enmarcados dentro del área Science Technology -ya que muchos de los resultados encontrados eran sobre tecnología y totalmente ajenos a esta investigación- la criba produce 21 resultados desde 1968 a 2015, no siendo ningún resultado de origen español. Los resultados, al igual que ocurría en Scopus, aunque tratan del método Doman, no se centran en el lenguaje, por lo que volvemos a filtrar la búsqueda con la palabra language y, finalmente, encontramos un único artículo (Von et al, 2013), en el que sí que se realiza una breve evaluación del lenguaje. En caso de realizar la misma búsqueda con las palabras clave en español, el resultado en las dos páginas web, utilizando "Método Doman", se reduce a un único resultado en Scopus, que es un artículo de fisioterapia y, en WOS, otro artículo que tampoco tiene relación directa con el Método Doman y el lenguaje.

Por todo lo referido, podemos confirmar que existe un vacío en la literatura científica sobre la evaluación del método Doman exclusivamente en lenguaje, que nos ayude a discernir y tener un juicio de valor probado por la comunidad científica sobre este tema. Por este motivo, creemos que este artículo es novedoso y puede arrojar luz sobre la utilización de los bits de inteligencia como una estrategia que ayuda a la inclusión de los niños con TDL en los contextos en los que está.

\section{1.-Intervención logopédica en retraso del lenguaje.}

No está demostrada cuál es la mejor intervención que se puede llevar con población infantil con trastornos del desarrollo del lenguaje (TDL). La clave para el éxito en la reeducación está basada en la globalización del tratamiento, es decir contemplar al niño con TDL en todas sus dimensiones no dividiéndolo en apartados estancos, y adaptar el método a cada uno de ellos individualizando el tratamiento.

En el CIE-11 (2018), podemos observar cómo los Trastornos del desarrollo del lenguaje se subdividen en trastornos del desarrollo con deficiencia del lenguaje receptivo y expresivo, del lenguaje expresivo, del lenguaje pragmático y con otro tipo de deficiencia específica del lenguaje.

Las características generales de esta población son la aparición tardía de palabras y estereotipos, ya que no aparecen hasta los 2-2.6 años y, cuando llegan a tener 3 años, no expresan más de cien palabras. La combinación de dos palabras se tarda otros seis meses o más en adquirir. También es lenta la evolución del nivel morfológico expresivo y la incorporación léxica expresiva. El habla en ocasiones está muy afectada, sin embargo, no es la dificultad prioritaria, la comprensión mejora en ambientes conocidos, pero no ocurre lo mismo en situaciones extrañas o complejas (Raventós, 2002).

La eficacia de la intervención lingüística en el aumento del léxico en niños con $R L$ y/o con TEL está altamente demostrada (e.g. Motsch y Ulrich, 2012; Moreno, Axpeo y Acosta, 2012), estos estudios utilizan diversos métodos para aumentar el léxico, pero ninguno de ellos utiliza los bits de inteligencia. Hay que tener en cuenta que el nivel semántico no es un nivel individual, sino que está adherido al resto de niveles, por lo tanto, tenemos que entender que los niños en general suelen mejorar de forma global 
con los tratamientos. En este caso, nuestro propósito en este estudio es emplear los bits de inteligencia con el fin de mejorar a nivel fonético y semántico principalmente, aunque no descartamos que paralelamente puedan mejorarse los otros niveles morfosintáctico y pragmático, como valoraremos posteriormente y, por consiguiente, ayudar a que estos niños estén mejor integrados en el contexto escolar.

\section{2.-Método.}

El enfoque cuantitativo es la característica básica de este estudio, lo cuantitativo como metodología de investigación es la utilización de los números para el estudio de la realidad natural y social. Esta investigación sigue un método de investigación cuantitativo preexperimental de medidas repetidas pre-post en el cual hay un único grupo (Campbell y Stanley, 2015).

La intervención con niños con retraso del lenguaje ha consistido en la aplicación de los bits de inteligencia empleando el PowerPoint como recurso. Para su creación se han seguido las pautas de Doman y Doman (1998).

\section{1.-Objetivos.}

La hipótesis que se persigue es que la aplicación del programa de los bits de inteligencia de Glenn Doman producirá en los participantes una mejora significativa en los diferentes componentes del lenguaje.

\section{1.-Población y Muestra.}

En el estudio que se presenta la muestra es intencional, formada en un principio por seis varones con retraso del lenguaje que acuden a sesiones individuales de 45 minutos de logopedia. Dos varones fueron excluidos al no realizar las evaluaciones finales y, por tanto, no concluir el tratamiento. Finalmente, la muestra se reduce a cuatro participantes.

Este artículo refleja un estudio piloto previo a la realización de un estudio mucho más amplio. Ese es el motivo por el que la muestra es tan reducida. El rango de edad está comprendido entre 55 y 79 meses, en la fecha de la evaluación inicial de cada uno de ellos, con una media de 70.25 meses $(\sigma=10.56)$. Son edades muy variadas que nos posibilitan observar la evolución y la respuesta en niños de diferentes edades.

Los criterios para la selección de la muestra son:

- Que sean niños y niñas diagnosticados de retraso del lenguaje.

- Que antes de comenzar este estudio ya acudieran a rehabilitación del lenguaje.

- Que se descarte previamente algún problema de pérdida auditiva, infecciones respiratorias y otitis media de repetición, que pudieran ser causantes del problema. 


\section{3.-Instrumentos.}

Se han seleccionado varios tipos de instrumentos, por un lado, un conjunto de cuestionarios y pruebas estandarizadas para evaluar el nivel del lenguaje de los participantes, y por otro, los bits de inteligencia elaborados ad hoc, empleando el PowerPoint como herramienta, y un cuestionario, también creado ad hoc, para conocer la opinión que tienen los padres.

Para llevar a cabo tanto la evaluación inicial como la evaluación final, se han utilizado las pruebas estandarizadas detalladas en la Tabla 1.

\section{Tabla 1}

\section{Pruebas estandarizadas}

\begin{tabular}{|c|c|c|}
\hline Test & Descripción & Objetivo de la prueba \\
\hline $\begin{array}{l}\text { El PLON-R. Prueba de Lenguaje Oral } \\
\text { Navarra- Revisada (Aguinaga, } \\
\text { Armentia, Fraile, Olangua y Uriz, } \\
\text { 1990). }\end{array}$ & $\begin{array}{l}\text { Esta prueba se divide en subpruebas: } \\
\text { Forma, Contenido y Uso. } \\
\text { La subprueba Forma incluye los } \\
\text { aspectos relacionados con los } \\
\text { aspectos formales, es decir, de la } \\
\text { topografía de la expresión verbal, sin } \\
\text { tener en cuenta los otros niveles } \\
\text { léxicos o pragmáticos. En esta } \\
\text { descripción se incluye la fonología, } \\
\text { morfología y la sintaxis. La subprueba } \\
\text { Contenido hace referencia a los } \\
\text { aspectos relacionados con el estudio } \\
\text { del significado de las palabras; es } \\
\text { decir, la semántica. } \\
\text { La subprueba Uso se incluye la } \\
\text { funcionalidad del lenguaje oral, es } \\
\text { decir, el nivel pragmático del lenguaje. }\end{array}$ & $\begin{array}{l}\text { Se utiliza con el objetivo de } \\
\text { evaluar el desarrollo del } \\
\text { lenguaje oral de los niños y, de } \\
\text { esta manera, comparar sus } \\
\text { resultados pre y postest. }\end{array}$ \\
\hline $\begin{array}{l}\text { El Registro Fonológico Inducido (RFI) } \\
\text { (Juárez y Monfort, 1996). }\end{array}$ & $\begin{array}{l}\text { Esta prueba incluye un material de } 57 \\
\text { tarjetas para la evaluación fonológica, } \\
\text { en expresión espontánea y en } \\
\text { repetición. }\end{array}$ & $\begin{array}{l}\text { Esta prueba nos aporta } \\
\text { información cuantitativa de los } \\
\text { aspectos fonológicos en } \\
\text { repetición y expresión } \\
\text { espontánea. }\end{array}$ \\
\hline $\begin{array}{l}\text { El Peabody Picture Vocabulary Test, } \\
\text { TVIP (Dunn, Dunn y Arribas, 2006). }\end{array}$ & $\begin{array}{l}\text { Evalúa el nivel de vocabulario } \\
\text { receptivo (nivel semántico). }\end{array}$ & $\begin{array}{l}\text { Los resultados de esta prueba } \\
\text { nos hacen conscientes de si } \\
\text { existe algún cambio en su nivel } \\
\text { de vocabulario, juntamente con } \\
\text { la subprueba de Contenido del } \\
\text { PLON. }\end{array}$ \\
\hline
\end{tabular}

Fuente: Elaboración propia

Instrumentos creados ad hoc para el estudio:

- $\quad$ Bits de Inteligencia de creación propia.

Se empleó la herramienta PowerPoint de Microsoft office 2010 para diseñar los bits de inteligencia siguiendo las líneas determinadas por Doman y Doman (1998). Cada PowerPoint está compuesto por 10 ítems, donde se incluyen 10 categorías semánticas, divididos en un total 21 PowerPoint. Se añadió voz a cada categoría semántica con el objetivo de homogeneizar la aplicación del programa, usando el mismo tono de voz y misma metodología con todos los niños. En la Tabla 2 se muestran las categorías y las palabras que forman parte del programa. 
Tabla 2

Vocabulario de los PowerPoint

\begin{tabular}{|c|c|c|}
\hline Categoría & $\begin{array}{l}N^{0} \text { de PowerPoint } \\
\text { de cada categoría }\end{array}$ & Palabras \\
\hline $\begin{array}{l}\text { Partes del } \\
\text { cuerpo }\end{array}$ & 2 & $\begin{array}{l}\text { Cabeza, boca, ceja, mejilla, barbilla, nariz, ojos, oreja, cuello, dientes, } \\
\text { frente, garganta, lengua, párpados, pestañas, brazo, codo, hombro, mano, } \\
\text { dedos, uña, pierna, dedos de los pies, muslo, pie, rodilla, tobillo, culo, } \\
\text { espalda y barriga. }\end{array}$ \\
\hline $\begin{array}{l}\text { Prendas de } \\
\text { vestir }\end{array}$ & 2 & $\begin{array}{l}\text { Abrigo, chaqueta, pantalón, jersey, camisa, falda, bufanda, gorro, } \\
\text { leotardos, botas, camiseta, chándal, zapatos, chanclas, calcetines, } \\
\text { calzoncillos, bragas, bañador, sombrero y playeras. }\end{array}$ \\
\hline Frutas & 2 & $\begin{array}{l}\text { Albaricoque, caqui, cereza, ciruela, fresa, granada, higo, kiwi, limón, } \\
\text { mandarina, mango, manzana, melocotón, melón, naranja, pera, piña, } \\
\text { plátano, sandía, y uva. }\end{array}$ \\
\hline Verduras & 2 & $\begin{array}{l}\text { Ajo, alcachofa, berenjena, seta, calabaza, cebolla, champiñón, maíz, } \\
\text { calabacín, zanahoria, coliflor, espárrago, guisante, judía, lechuga, patata, } \\
\text { pepino, pimiento, puerro y tomate. }\end{array}$ \\
\hline Carne & 1 & $\begin{array}{l}\text { Filete, hamburguesa, beicon, costilla, jamón, chorizo, salchichón, } \\
\text { mortadela, pollo, salchicha. }\end{array}$ \\
\hline $\begin{array}{l}\text { Otros } \\
\text { alimentos }\end{array}$ & 2 & $\begin{array}{l}\text { Pescado, pizza, cereales, magdalenas, bollo, tarta, patatas fritas, } \\
\text { gominolas, pepinillos, aceitunas, leche, batido, agua, zumo, Coca- cola, } \\
\text { yogurt, galletas. }\end{array}$ \\
\hline Objetos & 2 & $\begin{array}{l}\text { Puerta, ventana, silla, mesa, cama, cuchara, tenedor, cuchillo, plato, vaso, } \\
\text { servilleta, lámpara, televisión, cuento, juguete, parque, columpio, tobogán, } \\
\text { teléfono, casa, llaves y gafas. }\end{array}$ \\
\hline Animales & 3 & $\begin{array}{l}\text { Gallina, gallo, cerdo, vaca, caballo, burro, pollito, conejo, oveja, pato, } \\
\text { elefante, tigre, león, cebra, mono, serpiente, ciervo, oso, lobo, cocodrilo, } \\
\text { perro, gato, rana, mosca, pájaro, mariposa, jirafa, canguro, leopardo e } \\
\text { hipopótamo. }\end{array}$ \\
\hline Transportes & 2 & $\begin{array}{l}\text { Coche, autobús, avión, barco, bicicleta, camión, camioneta, moto, } \\
\text { furgoneta, globo aerostático, helicóptero, lancha motora, metro, monopatín, } \\
\text { motocicleta, moto de agua, patines, patinete, taxi y tren. }\end{array}$ \\
\hline Opuestos & 2 & $\begin{array}{l}\text { Día-noche, frío-calor, encima-debajo, dentro-fuera, llena-vacía, limpia- } \\
\text { sucia, lento-rápido, largo- corto, joven-viejo y feliz-triste. }\end{array}$ \\
\hline
\end{tabular}

Fuente: Elaboración propia

- Cuestionario para las familias

También se ha elaborado un cuestionario para conocer la opinión de las familias sobre el método. El número de cuestionario que se aplicaron fueron cuatro cuestionarios, uno a cada familia.

El cuestionario consiste en una escala tipo Likert de 10 puntos, en el que (1) representa la puntuación más baja/lo menos frecuente, y el (10) la máxima frecuencia. El cuestionario consta de 10 ítems (p.e. "Conocía de forma previa a la utilización del método las palabras expuestas") donde los familiares tienen que marcar con una X la frecuencia que consideren más apropiada. El cuestionario se lleva a cabo durante la última sesión de intervención, cuando finaliza la sesión de sus hijos. El cuestionario valora la importancia que atribuyen los familiares a los especialistas del lenguaje, la eficacia del método, la frecuencia con la que aplican los bits en casa y la estimación de la mejora que encuentran en sus hijos. 


\section{4.-Procedimiento de recogida y análisis de datos.}

La intervención logopédica empleando los bits de inteligencia adaptados del método Glen Doman, se lleva a cabo durante un mínimo de 9 semanas y un máximo de 15 semanas, en 3 fases: Fase 1: evaluación inicial, Fase 2; rehabilitación del lenguaje y Fase 3: evaluación final.

Fase 1. Evaluación inicial: en esta fase se les explican a las familias las tres pruebas estandarizadas (descritas en el apartado de instrumentos) que se les van a pasar a sus hijos y cómo va a ser el proceso de aplicación, que será de manera individual en la sala de intervención logopédica. También se les explica que tienen la posibilidad paralelamente de ir aplicando los bits en casa y cómo llevarlo a cabo. Ellos deben aceptar las condiciones del servicio, firmando un consentimiento informado. Se evalúa al participante en una primera sesión de una hora de duración, la primera prueba que se aplica es el PLON-R, después el PEABODY, y finalmente el RFI.

Fase 2. Rehabilitación del lenguaje: esta fase se centra en la puesta en marcha del programa adaptado de Glenn Doman con los bits de inteligencia. Las familias participarán de forma activa en la intervención. Se les explica que tienen que mostrar a sus hijos en casa las diapositivas una vez al día, aconsejándoles que lo realicen precisamente los días que no acudan a la sesión, puesto que los días que el niño acude a la sesión lo hará la logopeda. Esta posibilidad no será obligatoria, evitando dar a la familia más responsabilidad de la que quieran o sean capaces de desarrollar. La comunicación con la familia será continua, directa y muy personal, siendo un pilar angular en nuestra intervención.

La sesión comienza mostrando a los niños las láminas, esta actividad dura alrededor de 1 minuto, en caso de que la motivación o la atención no sean adecuadas en ese momento, se podrá posponer.

Durante una semana se muestra a los niños el mismo PowerPoint. Como los niños acuden con el logopeda dos sesiones semanales, los otros cinco días será la familia la encargada de mostrarles las láminas en casa. Esta tarea se realiza durante las semanas que dura el programa con cada niño.

Fase 3: Evaluación final: en esta fase se vuelven a aplicar las tres pruebas estandarizadas que se pasaron en la Fase 1, con la finalidad de comprobar si los niños han mejorado en el nivel fonológico-fonético, semántico y pragmático. En esta misma fase, y una vez finalizada la intervención, se les presenta a los padres el cuestionario para que valoren el proceso llevado a cabo.

Los datos obtenidos se han tratado con el paquete estadístico SPSS 24.0, para Windows. En todas las pruebas se establece un nivel de confianza del 95\%, se han establecido frecuencias, medias y porcentajes según cada variable.

Se recoge la Puntuación Típica (PT) del Peabody y del PLON-R, y se recoge la Puntuación Directa (PD) del RFI en expresión espontánea, no teniendo en cuenta la expresión repetida.

Además de realizarse los estadísticos descriptivos, las pruebas que se han realizado fueron la Prueba T-Student y la correlación de Pearson. La Prueba T-Student se utiliza para muestras relacionadas en algunas variables y nos ayuda a observar la mejoría de los participantes en las diferentes pruebas. 
También realizamos la correlación de Pearson para observar el nivel de covariación entre las mismas pruebas, realizadas antes y después de la intervención, para encontrar una posible relación lineal entre ellas.

\section{3.-Resultados.}

Uno de los primeros resultados que encontramos es la diferencia existente en el tiempo máximo al que acudieron a las sesiones. Podemos observar estos datos en la figura 1. Los cuatro niños utilizan los bits en las sesiones con la logopeda y de forma complementaria las familias aplican los bits en casa únicamente con los niños 1 y 4 , sombreados en las columnas de color azul; paradójicamente estos mismos niños son los que menos tiempo de intervención en general han tenido unas 9 semanas. Sin embargo, los participantes 2 y 3 no han utilizado los bits en casa, pero han recibido en general mayor tiempo de intervención. Los participantes han cooperado en el procedimiento durante un mínimo de 9 semanas y un máximo de 15 semanas. Han utilizado los bits de inteligencia en las sesiones de intervención logopédica y en casa durante una media de 12 semanas $(\sigma=2.94)$.

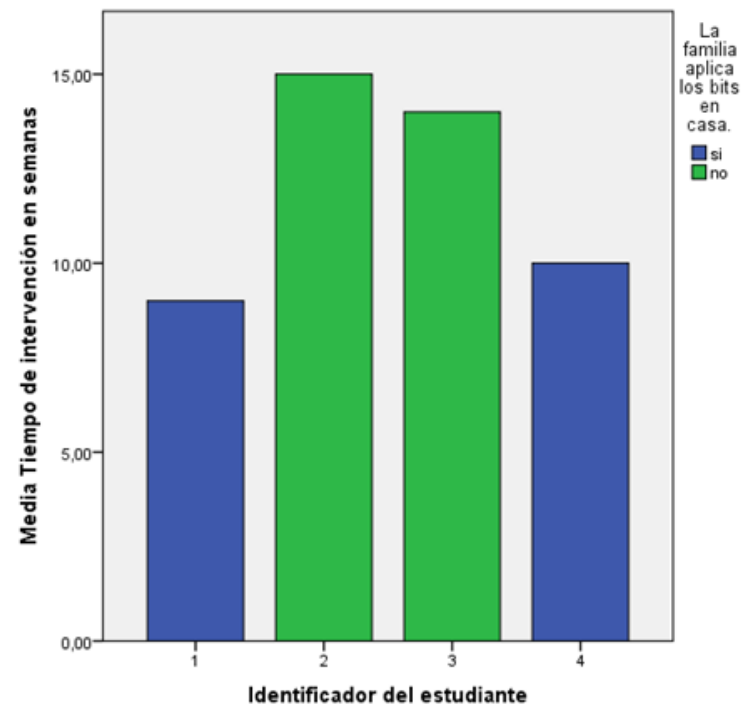

Figura 1: Relación del tiempo de intervención por cada caso, y la aplicación de los bits en casa. Elaboración propia

Los resultados obtenidos de las pruebas estandarizadas evidencian la normalidad de las variables con Shapiro Wilk, determinando que nuestros valores provienen de una distribución normal.

La comparación de las puntuaciones medias entre el pretest y el postest se realizó a través de la Prueba comparativa T de Student para muestras relacionadas, mostró que la mejora del nivel fonético-fonológico fue significativa, mientras que no fue significativa en el resto de las variables. Cuando el nivel Sig (bilateral) es menos o igual a 0.05 se 
acepta $\mathrm{H} 1$ por lo que la aplicación del programa adaptado producirá en los participantes una mejora significativa. Solamente encontramos un resultado que cumpla estas características, es el último dato de la tabla 3 que está escrito en negrita. En la tabla 4 también se resaltan estos resultados en negrita.

Tabla 3.

Prueba de muestras emparejadas

\begin{tabular}{|c|c|c|c|c|c|c|c|}
\hline & & & & & & $T$ & $\begin{array}{c}g l \\
\text { Sig. (bilateral) }\end{array}$ \\
\hline & Media & $\begin{array}{l}\text { Desviación } \\
\text { estándar }\end{array}$ & $\begin{array}{l}\text { Media de } \\
\text { error } \\
\text { estándar }\end{array}$ & $\begin{array}{l}95 \% \text { de ir } \\
\text { confianza } \\
\text { diferencia } \\
\text { Inferior }\end{array}$ & $\begin{array}{l}\text { intervalo de } \\
\text { de la } \\
\text { Superior }\end{array}$ & & \\
\hline $\begin{array}{l}\text { Correlación entre } \\
\text { PLON Forma pre y } \\
\text { post test }\end{array}$ & -2.5 & 11.09054 & 5.54527 & -20.14752 & 15.14752 & -.451 & .683 \\
\hline $\begin{array}{l}\text { Correlación entre } \\
\text { PLON Contenido } \\
\text { prey yost test }\end{array}$ & -9 & 9.273618 & 4.636809 & -23.756396 & 5.756396 & -1.941 & .148 \\
\hline $\begin{array}{l}\text { Correlación entre } \\
\text { PLON uso pre y } \\
\text { post test }\end{array}$ & -1.75 & 3.50000 & 1.75000 & -7.31928 & 3.81928 & -1.000 & .391 \\
\hline $\begin{array}{l}\text { Correlación entre } \\
\text { PLON total pre y } \\
\text { post test }\end{array}$ & -4.5 & 7.93725 & 3.96863 & -17.12994 & 8.12994 & -1.134 & .339 \\
\hline $\begin{array}{l}\text { Correlación entre el } \\
\text { Peabody pre y post } \\
\text { test }\end{array}$ & -4.75 & 5.12348 & 2.56174 & -12.90259 & 3.40259 & -1.854 & .161 \\
\hline $\begin{array}{l}\text { Correlación entre el } \\
\text { RFI pre y post test }\end{array}$ & 26.5 & 4.43471 & 2.21736 & 19.44338 & 33.55662 & 11.951 & .001 \\
\hline
\end{tabular}

Fuente: Elaboración propia

Los resultados nos muestran que son similares en los cinco primeros pares: los resultados totales del PLON (P-Valor $0.339>0.05)$ y las subpruebas de esta misma prueba: Forma (P-Valor $0.683>0.05)$, Contenido (P-Valor $0.148>0.05)$, y Uso (PValor $0.391>0.05)$; también ocurre con la prueba de Peabody ( $P$-Valor $0.161>0.05)$. En todos estos casos, al ser mayor el valor que 0.05 , tenemos que rechazar nuestra hipótesis y afirmar que no hay una diferencia en las medidas de las pruebas antes y después de la intervención. Sin embargo, sí que existen diferencias entre la media ponderada previa y posterior de cada una de las pruebas, pero dicha media no es significativa.

Por otro lado, como se puede observar en la Tabla 4 los resultados del RFI (P-Valor $\leq$ 0.001 ) sí que cumplen los requisitos para aceptar nuestra hipótesis, es decir, se puede afirmar que hay una diferencia significativa en las medidas de las pruebas antes y después de la intervención en el Registro Fonológico Inducido, que evaluaba el nivel fonológico-fonético de los participantes. Cuando realizamos el estadístico descriptivo de ambas pruebas observamos que en la evaluación inicial en el RFI los niños muestran una media de 56 errores, mientras que en la evaluación final muestran una media de 29.5 errores. Por lo que encontramos una diferencia menor en la media ponderada de 26.50 entre la primera prueba de RFI y la segunda. 
Tabla 4.

Correlación de Pearson del Registro Fonológico Inducido

\begin{tabular}{|c|c|c|c|}
\hline & & $\begin{array}{l}\text { RFI. E } \\
\text { inicial }\end{array}$ & $\begin{array}{l}\text { IRFI. Evaluación } \\
\text { final }\end{array}$ \\
\hline $\begin{array}{l}\text { RFI. Evaluación inicial. Total de } \\
\text { fonemas erróneos }\end{array}$ & $\begin{array}{l}\text { Correlación de Pearson } \\
\text { Sig. (bilateral) } \\
\text { N }\end{array}$ & 1 & $\begin{array}{l}.977^{*} \\
.023 \\
4\end{array}$ \\
\hline $\begin{array}{l}\text { RFI. Evaluación final. Total de } \\
\text { fonemas erróneos }\end{array}$ & $\begin{array}{l}\text { Correlación de Pearson } \\
\text { Sig. (bilateral) } \\
\text { N }\end{array}$ & $\begin{array}{l}.977^{*} \\
.023 \\
4\end{array}$ & 1 \\
\hline \multicolumn{4}{|c|}{ * La correlación es significativa en el nivel 0.05 (bilateral) } \\
\hline
\end{tabular}

Fuente: Elaboración propia

En el análisis de correlación de Pearson del RFI, (Tabla 4) vislumbramos que la correlación es significativa ya que p valor (sig, bilateral) $0.023<0.05$, por lo que hay una correlación positiva entre las dos pruebas, algo que no ocurre entre las anteriores. A través de la correlación de Pearson, también se realizaron otro tipo de correlaciones como la relación que existe entre el tiempo de intervención en semanas y los datos de la evaluación final de Peabody, que han sido positivos ( $p$ valor $0.001<0.05$ ), al igual que la correlación positiva entre el puntaje del Peabody al inicio del tratamiento y la subprueba Contenido del PLON al inicio del tratamiento ( $p$ valor $0.05=0.05$ ). Existen más correlaciones positivas entre pruebas como la subprueba Contenido del PLON al inicio del tratamiento y los resultados iniciales del RFI ( $p$ valor $0.45<0.05$ ). Lo mismo ocurre con los resultados iniciales y finales de la subprueba Uso del PLON ( $p$ valor $0.33<0.05)$ y los resultados iniciales y finales total del PLON, que tienen una correlación de $p$ valor $0.045<0.05$.

Por otro lado, los resultados obtenidos de los cuestionarios (Gráfico 2) nos muestran lo siguiente:

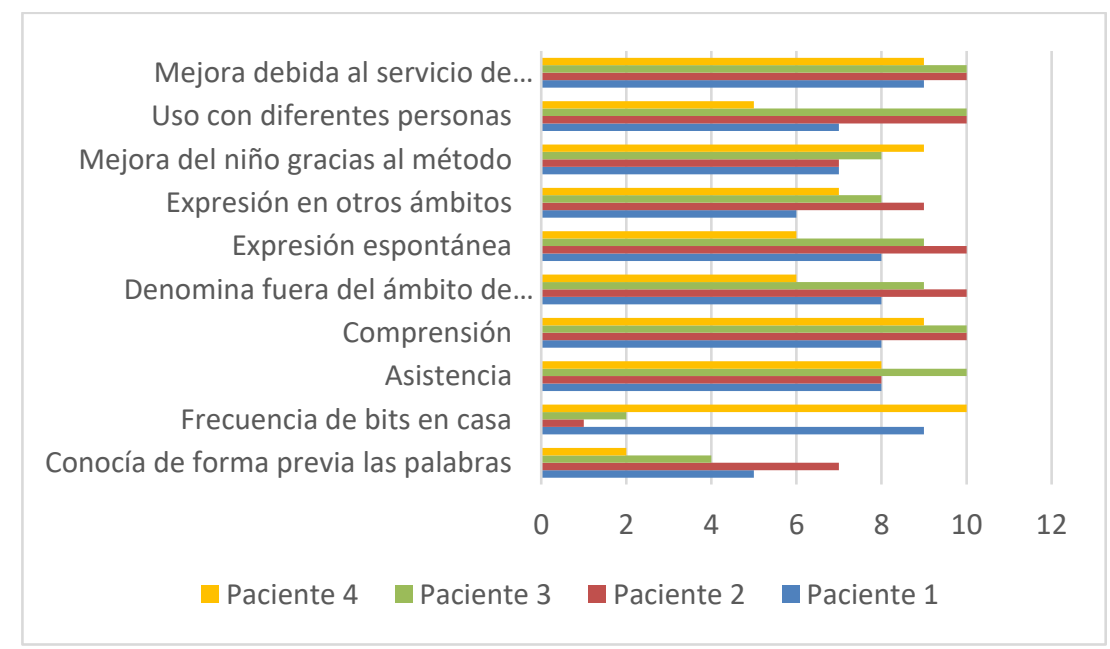

Figura 2. Cuestionario para las familias. Elaboración propia 
Así pues, ante la pregunta del cuestionario que se encuentra en la figura 2 ¿Conocía de forma previa a la utilización del método las palabras expuestas?, la media de las palabras que los padres consideraban que sus hijos conocían anteriormente es de 4.5, por lo que la mayoría de las palabras que aparecen en los PowerPoint no son conocidas. Pero es cierto que un $75 \%$ de las familias hacen referencia a que esas categorías semánticas se han trabajado en la Escuela Infantil a la que acudían los niños.

Respecto a la cuestión frecuencia de aplicación de los bits en casa, los datos nos muestran una desviación estándar muy alta $(\sigma=4.65)$, que es debido a la diferencia entre los resultados, ya que el $50 \%$ de las familias no participaron en la aplicación de los bits en casa.

Respecto a la asistencia, la media es de 8.50. Teniendo en cuenta que la última parte de la intervención se realizó en verano, estimamos que la asistencia ha sido bastante alta.

La media de la comprensión de las palabras en distintos ámbitos (cuarto ítem) es muy cercana a 10 (9.25) con una desviación típica baja ( $\sigma=9.57$ ), lo que significa que las respuestas de los participantes fueron similares.

Si comparamos la media de las respuestas del apartado de comprensión (9.25) con los de la expresión espontánea de las palabras (8.25) y la expresión de las palabras en diferentes ámbitos (8.25), comprobamos que es menor la percepción de la mejora en expresión que en comprensión por parte de las familias; es decir, creen que los niños han mejorado más en la comprensión que en la expresión. Y la valoración de la expresión de estas palabras en otros ámbitos (7.50) es aún más baja que el del resto de ítems; es decir, los niños han aprendido las palabras y las utilizan en su ámbito más cercano, pero la familia no cree que las utilicen en otros ámbitos o con otras personas. A su vez, llama la atención la diferencia en la media ponderada entre la mejoría que atribuye la familia al método (7.75), respecto a su opinión sobre la intervención logopédica en general (9.50), esto quiere decir que tienen una mejor opinión sobre la intervención en general que sobre el método en particular.

\section{4.-Discusión y conclusiones.}

Tal y como se deduce de todo lo comentado, podemos señalar que este estudio acepta el uso de los bits de inteligencia en niños con TDL con la finalidad de mejorar su nivel fonológico y fonético; pero se rechaza su utilización, tal y como Doman lo propuso, con el objetivo de mejorar los niveles morfosintáctico, semántico y/o pragmático del lenguaje. Por tanto, admitimos nuestra hipótesis en el nivel fonológico y fonético; es decir, la aplicación del programa de los bits de inteligencia de Glenn Doman producirá en los participantes una mejora significativa en nivel fonológico y fonético, y rechazamos nuestra hipótesis en el resto de los componentes del lenguaje (semántico, morfosintáctico y pragmático); así pues, la aplicación del programa de los bits de inteligencia de Glenn Doman no producirá en los participantes una mejora significativa en estos niveles.

Respecto a la intervención de las familias en el tratamiento consideramos que es necesaria. En nuestro estudio las familias muestran un grado de satisfacción notable con el método, $(x=7.75)$, aunque en general es mayor el grado de satisfacción en la 
intervención logopédica $(x=9.50)$. Esto puede deberse a la escasa mejoría que han mostrado, una vez seguido el método, y al conocimiento de las palabras con anterioridad a la aplicación del método $(x=8)$. También es llamativa la baja aplicación de los bits en el entorno natural ( $x=5.5)$, esto es debido a que el $50 \%$ de las familias se negaron a aplicar los bits en casa por el hecho de tener que utilizar el ordenador con el PowerPoint.

En los estudios de Moreno et al., (2012) y Motsch y Ulrich (2012), encontramos que los niños con trastornos del lenguaje mejoran en el nivel semántico con rehabilitación. En cambio, en nuestro estudio hemos comprobado que, ni en los resultados parciales ni totales del PLON ni en el Peabody, hubo una mejora significativa en el nivel semántico. Por lo que no se corresponden los resultados encontrados en Moreno et al., (2012) y Motsch y Ultrich (2012), con los de nuestro estudio.

Para comprender nuestros resultados debemos tener en cuenta lo que medía cada apartado de cada prueba. La medida PLON Forma indicaba el nivel fonológico y morfosintáctico, como un único ítem; es decir que, aunque los niños no obtuvieran una mejora significativa en esta subprueba conjunta, sí que lo tienen si evaluamos por separado el nivel fonológico, como se hizo posteriormente con el RFI ya que se obtuvo mucha mejoría, en la evaluación inicial del RFI los participantes mostraron una media de 56 errores, mientras que en la evaluación final mostraron una media de 29.5 errores. De forma parecida ocurre con la medida de PLON Contenido y el Peabody, ambos analizaban el nivel semántico, la medida de PLON Uso observaba el nivel pragmático y en ninguno se obtuvo una mejora significativa.

Por lo que, teniendo en cuenta todo ello, podemos concluir que el uso de los bits de inteligencia en esta población no hace que los niños con TDL mejoren significativamente en estas áreas morfosintácticas, semánticas y pragmáticas.

Motsch y Ulrich (2012), en su estudio, no encuentran una relación significativa entre la mejora en el volumen del vocabulario expresivo y la memoria fonológica a corto plazo; lo mismo ocurrió en nuestro estudio, ya que los niños no han mejorado significativamente en los niveles morfosintáctico, semántico ni pragmático, pero sí en los niveles fonológico-fonético. Este hecho se aprecia en el RFI, en el que los niños sí que mostraron una mejora significativa. El RFI mide exclusivamente el nivel fonéticofonológico, por lo que podemos concluir que el uso de los bits de inteligencia sí que hace que los niños mejoren en estos dos niveles. Lo que por siguiente se puede traducir en una mayor inclusión escolar, y la reducción de los problemas académicos que estos niños suelen mostrar.

Si comparamos los resultados con los del estudio de Von et al. (2013), nuestros resultados son muy inferiores a los suyos. Puede deberse a que el tiempo de intervención que ellos utilizaron fue de dos años y el nuestro ha sido muy inferior entre 9 y 15 semanas- por lo que también es comprensible que la ganancia media en la comprensión del lenguaje fue de ocho meses, mientras que en nuestro estudio no se ha observado esa mejoría. Tengamos en cuenta que es difícil establecer esta comparativa, pues tampoco se trata de la misma población, ya que la población de dicho estudio eran niños con parálisis cerebral, síndromes genéticos, retraso del desarrollo, daño cerebral adquirido y niños con epilepsia. Por ello, no podemos esperar que los resultados obtenidos con nuestros niños con TDL mejoren de la misma manera, aunque el método sea el mismo. 
Insistimos finalmente en que, como ya hemos comentado, no existen investigaciones científicas que avalen la eficacia de los bits en niños y niñas con retraso del lenguaje, por lo que no podemos comparar con otros estudios. Aunque, teniendo en cuenta nuestros datos consideramos que podría existir una mejora en el nivel semántico de los niños con TDL, realizando unas recomendaciones y adaptaciones del método. Estas propuestas son consecuencia del proceso de intervención y de las opiniones de las familias. Dichas adaptaciones son:

- Seleccionar unos bits de inteligencia según su frecuencia de uso (no enmarcados dentro de unas categorías semánticas).

- Variar el soporte de aplicación utilizando un soporte con acceso a internet, facilitando a los padres una aplicación más sencilla. Se sugiere usar desde un soporte tipo "Nube" en el que subamos un video, o hasta aplicaciones como "Youtube".

- Ampliar el número de diapositivas / bits de inteligencia que se enseñan a los niños por día, ya que las propuestas de mejora señalaban como "muy bajo" el número de ítems que se mostraban al día.

- Diseñar estrategias para animar a las familias a aplicar los bits de inteligencia en casa y de esta manera aumentar la estimulación.

Consideramos importante continuar con esta línea de investigación puesto que se trata de un método muy empleado en el ámbito logopédico. Puede ser un método para utilizar tanto de forma preventiva para la población infantil como para la intervención educativa en niños/as con retraso del lenguaje. Para ello, es necesario encontrar evidencias científicas claras y contundentes que nos ayuden a tomar la decisión sobre la efectividad del uso de los bits de inteligencia del método Doman en la intervención lingüística. Es fundamental realizar estudios similares aumentando el tiempo de intervención y con un mayor número de participantes.

\section{5.-Referencias.}

American Academy for Cerebral Palsy, American Academy of Neurology, American Academy of Pediatrics, American Academy for Physical Medicine and Rehabilitation, American Congress of Rehabilitation Medicine, American Academy of Orthopedics..., National Association for Retarded Children (USA) (1968). The Doman-Delacato Treatment of Neurologically Handicapped Children. Developmental Medicine and Child. Neurology, 10, 243-246.

American Academy of Pediatrics. (1999). The treatment of neurologically impaired children using patterning. Pediatrics, 104(5), 1149-1151.

Campbell, D.T., Stanley, J.C. (2015). Experimental and quasi-experimental designs for research. U.S.A: Ravenio Books.

Doman, G., Doman, J., Aisen, S. (2012). Cómo enseñar conocimientos enciclopédicos a su bebé. La Revolución Pacífica. Madrid: Edaf. 
Doman, G., Doman, J. (1998). Cómo multiplicar la inteligencia de su bebé: la revolución pacífica (Vol. 7). Madrid: Edaf.

Moreno, A., Axpe, Á., Acosta, V. (2012). Efectos de un programa de intervención en el lenguaje sobre el desarrollo del léxico y del procesamiento fonológico en escolares de Educación Infantil con Trastorno Específico del Lenguaje. Revista de Investigación Educativa, 30 (1), 71-86

Motsch, H.J., Ulrich, T. (2012). Effects of the strategy therapy 'lexicon pirate' on lexical deficits in preschool age: A randomized controlled trial. Child Language Teaching and Therapy, 28(2), 159-175. https://doi.org/10.1177/0265659011432943

Raventós, M.S. (2002). Trastornos del lenguaje: preguntas pendientes en investigación e intervención. Revista de Logopedia, Foniatría y Audiología, 22(2), 63-76. https://doi.org/10.1016/S0214-4603(02)76224-4

Von, S., Verdel, M., Barstad, B. G., Gravås, E. M., Jahnsen, R., Krabbe, S., ... \& Bang, B. (2013). The effect of interventions based on the programs of The Institutes for the Achievement of Human Potential and Family Hope Center. Developmental neurorehabilitation, 16(4), 217-229. https://doi.org/10.3109/17518423.2012.739211

World Health Organization. (2019, May 15). ICD-11. Geneva, Switzerland: ICD-11 for Mortality and Morbidity Statistics(11th Revision) https://icd.who.int/browse11/l-m/en 